\title{
Zur Glazialproblematik auf Blatt Freiburg-Süd der Geomorphologischen Karte 1:100000 der Bundesrepublik Deutschland (GMK 100, Blatt 2)
}

\author{
HARTMUT LESER *)
}

\author{
Middle Pleistocene, Riß glaciation, glacier, Alpine Ice, \\ glacial morphology, geomorphological mapping \\ Black Forest, Wehra Valley, Dinkelberg, Baden-Württemberg \\ TK 25: Nr. 8313; TK 100: Nr. C 8310
}

Kurzfassung: Der Artikel bezieht sich auf Kartierungen des Blattes Freiburg-Süd der GMK 100, die ihren Ausgang in der Aufnahme des Blattes Wehr der GMK 25 nahm. Große Teile des Blattes Freiburg-Süd gelten nach herrschenden Vorstellungen während der Riß-Kaltzeit als vergletschert. Die Kartierungen, besonders an der Nahtstelle zwischen Alpen- und Schwarzwaldeis (Mündung des Wehratales in den Hochrhein; Hochrheintal), beweisen den Eiskontakt. Das Schwarzwaldeis war jedoch auf das Wehratal beschränkt. Dieser Talgletscher erreichte nicht die Höhen des Dinkelberges. Eine ausgedehnte Eisüberdeckung des Dinkelberges ist nach den bisherigen Befunden wenig wahrscheinlich.

[Glacial Problems at sheet Freiburg-Süd of the Geomorphological Map 1:100000 of the Federal Republic of Germany (GMK 100, Sheet 2)]

Abstract: This article is based on the mapping of sheet Freiburg-Süd of the GMK 100, which has been started by mapping of sheet Wehr of the GMK 25. In the past the suggestion existed, that large areas of the sheet Freiburg-Süd have been glaciated during the Riss glaciation. The mappings furnished the proof of the ice mass contact between both Alpine ice and Black Forest ice, especially at the mouth

*) Anschrift des Autors: Professor Dr. rer. nat. H. LESER, Forschungsgruppe Geomorphologie, Geographisches Institut der Universität Basel, Klingelbergstr. 16, $\mathrm{CH}-4056$ Basel/Schweiz.

Der Verfasser dankt der Deutschen Forschungsgemeinschaft für die Möglichkeit, daß er im Rahmen des Schwerpunktprogramms "Geomorphologische Detailkartierung in der Bundesrepublik Deutschland" als Kartierer und Mitglied der Koordinationskommission mitwirken durfte. Er dankt den Kollegen von der Koordinationskommission (Prof. BARSCH - Heidelberg, Prof. FränZle - Kiel, Prof. LIEDTKE - Bochum und Prof. STÄBLEIN - Bremen) für dauernde Gesprächs- und Diskussionsbereitschaft. of the Wehra valley into the High Rhine valley. The Black Forest ice was restricted to the Wehra valley. This valley glacier did not reach the higher parts of the Dinkelberg. According to recent findings an extended ice cover on the Dinkelberg is most unlikely.

\section{Einleitung}

Im Rahmen des GMK-Schwerpunktprogrammes der DFG wurde zunächst das Blatt Wehr (GMK 25 Blatt 4; TK 25 Blatt 8313) kartiert (LESER 1979a, 1979b), Im Rahmen verschiedener geomorphogenetischer Folgearbeiten (LESER 1980, 1981 a, 1981 b) wurde die Glazialproblematik des Würm und des Riß verfolgt. Dies geschah auch für das angrenzende Möhliner Feld (Schweiz) durch eine Mitarbeiterin (KÜHNEN 1983, 1984). Die parallel laufende Kartierung des Blattes Freiburg-Süd der GMK 100 (LESER 1985a, 1985 b) gab Anlaß, die Riß-Vereisungsproblematik des Schwarzwald-Südrandes und im Bereich des Eiskontaktes im Hochrheintal, an der Wehramündung, größerräumig zu verfolgen. Darüber wird im Zusammenhang ausführlich und an anderer Stelle berichtet (LESER \& MeTz [o. J.; im Druck]).

Der Verfasser kommt zu der Auffassung, daß die von PFANNENSTIEL \& RAHM $(1964,1975)$ dargestellte rißzeitliche Eisverbreitung zumindest im Bereich des Dinkelberges mit Vorsicht gehandhabt werden muß. Auch die verdienstvollen Arbeiten von RAHM (1970, 1980) und von HANTKE (1978) bringen für eine vollständige Vereisung des Dinkelberges, wie sie in den Arbeiten dieser Autoren immer wieder dargestellt wird und die letztlich auf der Vorstellung bei PFANNENSTIEL \& RAHM (1964) basiert, keinen überzeugenden Beweis. Die geomorphologische Kartierung und 
die Substrataufnahme auf Blatt Wehr der GMK 25 und Freiburg-Süd der GMK 100 ließen in Formenschatz und Material eindeutige Glazialhinweise vermissen. Daraufhin versuchte der Verfasser, eine neue Hypothese für die Ausbildung des Einrandes am Südschwarzwald - während der Riß-Kaltzeit - zu geben. Darauf beziehen sich nachstehende Überlegungen, die in Beziehung zu dem in der farbigen Kartenbeilage dargestellten Formenschatz zu setzen wären. Auch die Hypothesen der anderen o. a. Autoren sollten in Beziehung zur geomorphologischen Bestandsaufnahme gesetzt werden.

\section{Methodik und Problem}

Die in Kap. 3 dargestellten Hypothesen basieren auf einer gründlichen Geländekenntnis, die im Rahmen geomorphologischer Kartierungsarbeiten gewonnen wurde. Dabei stand, neben der geomorphographischen Aufnahme nach den GMK 25- und GMK 100Legenden (LESER \& STÄBLEIN 1978; FRÄNZIE et al. 1979), die Aufnahme geomorphogenetischer Substrattypen im Mittelpunkt der Arbeit. Sie finden sich in den beiden GMK-Blättern des Gebietes (FreiburgSüd und Wehr) dargestellt. Die Aufnahme erfolgte auf GMK 25-Blatt Wehr im Rahmen einer Punkt für Punkt durchgeführten flächendeckenden Begehung. Kaum ein Punkt des Blattes Wehr dürfte nicht direkt eingesehen worden sein. Die Kartierung des GMK100-Blattes Freiburg-Süd erfolgte in einer dem Maßstab (und damit der Gebietsgröße) angemessenen Arbeitsweise. Darüber geben auch die beiden Erläuterungshefte (LESER 1979 b, 1985 b) Auskunft.

Der Hinweis auf diese Methodik (zu der noch einschlägige Aufschlußaufnahmen gehörten) erfolgt deswegen, weil bei der Kartierung an sich alle wesentlichen Glazialspuren erkannt sein müßten. Speziell für Blatt Wehr, dem äußersten SE-Sechzehntel des Blattes Freiburg-Süd, mit zugleich dem entscheidenden Nahtbereich zum Alpeneis im Hochrheintal, konnten gewisse Belege für die Riß-Kaltzeit erbracht werden. Darüberhinausgehende Spuren wurden nicht entdeckt. Die Anschlußbearbeitung erfolgte auf dem Möhliner Feld durch KÜHNEN $(1983,1984)$ unter Anleitung des Verfassers. Aus dem Zusammenhang der Gebietserkenntnisse und deren Einpassungen in den räumlich größeren Rahmen des Blattes FreiburgSüd der GMK 100 resultieren die in Kap. 3 und den dazugehörigen Abbildungen dargestellten Hypothesen.

\section{Hypothesen zur Ausdehnung des rißzeitlichen Schwarzwaldeises nach S und der Kontakt zum Alpeneis des Rheingletschers im Hochrheintal}

Die Basishypothese wird in Abb. 1 dargestellt. Sie beruht auf den Kartiererfahrungen, daß

- der Formenkontext nicht ausreicht, eine glaziale Überformung des gesamten Dinkelberges anzunehmen und daß dort

- glazigene Sedimente in einem einwandfreien stratigraphischen Zusammenhang fehlen. (Der umstrittenen Schotterstreu konnte der Verfasser keine Beweiskraft abgewinnen.)

Daraus leiten sich folgende Setzungen ab:

- Eine Vorla ndverglets cherung auf der Schwarzwald-Südseite kann es während der Riß-Kaltzeit allenfalls in einem ganz beschränkten Umfang gegeben haben. Siehe dazu Abb. 1 und Erläuterungen.

- Sie ist belegt für das eigentliche Wehratal und seine Ränder, das e i s e $\mathrm{r} f$ ü $1 \mathrm{l} \mathrm{t}$ gewesen sein muß. Sie ist nicht belegt für die Höhen des Dinkelberges, der sich nur wenig, aber für eine geringmächtige Eismasse zu bedeutsam, über das Wehratal erhebt. Siehe dazu Abb. 2 und Erläuterungen.

- Den E is k o n t a k t zwischen Schwarzwaldund Alpeneis, also dem Wehra- und dem Rheingletscher, kann man mit hoher Sicherheit annehmen, weil sowohl durch PfanNENSTIEL \& RAHM (1964; vom Verfasser ebenfalls nachgewiesen) als auch noch weiter südlich, in Öflingen (LESER 1981b), Grundmoräne a u f Flußterrasse nachgewiesen wurde. Dies also in unmittelbarer Nähe des Hochrheintales. Siehe dazu Abb. 3 und 4 und Erläuterungen.

Zur ersten $\mathrm{H}$ y p ot hese: Der Formenschatz auf GMK 100-Blatt Freiburg-Süd zeigt, daß sich entlang des gesamten Nordrandes des Dinkelberges $\mathrm{ke}$ in erlei $\mathrm{Hinw}$ e is e a f G la$\mathrm{z}$ i a l s p u r e $\mathrm{n}$ erkennen lassen. Eine Eisüberfahrung kann nicht spurlos an den morphologisch weichen Kalkgesteinen vorübergegangen sein. Selbst wenn man eine auch nur randliche Eisüberformung des Dinkelberges annehmen würde, wären - wegen der lösungskräftigen, $\mathrm{CO}_{2}$-haltigen Schmelzwässer zumindest Relikte von Flie $B$ ge wä s se $r$ forme $n$ zu erwarten, die auf das glaziale Hinterland hätten hinweisen müssen. Die eindeutig ausgebildeten rißzeitlichen $\mathrm{Flu} \mathrm{Bt}$ e $\mathrm{r}$ a s s e $\mathrm{n}$ $\mathrm{n}$ i v e a u am nördlichen Dinkelbergrand kann 


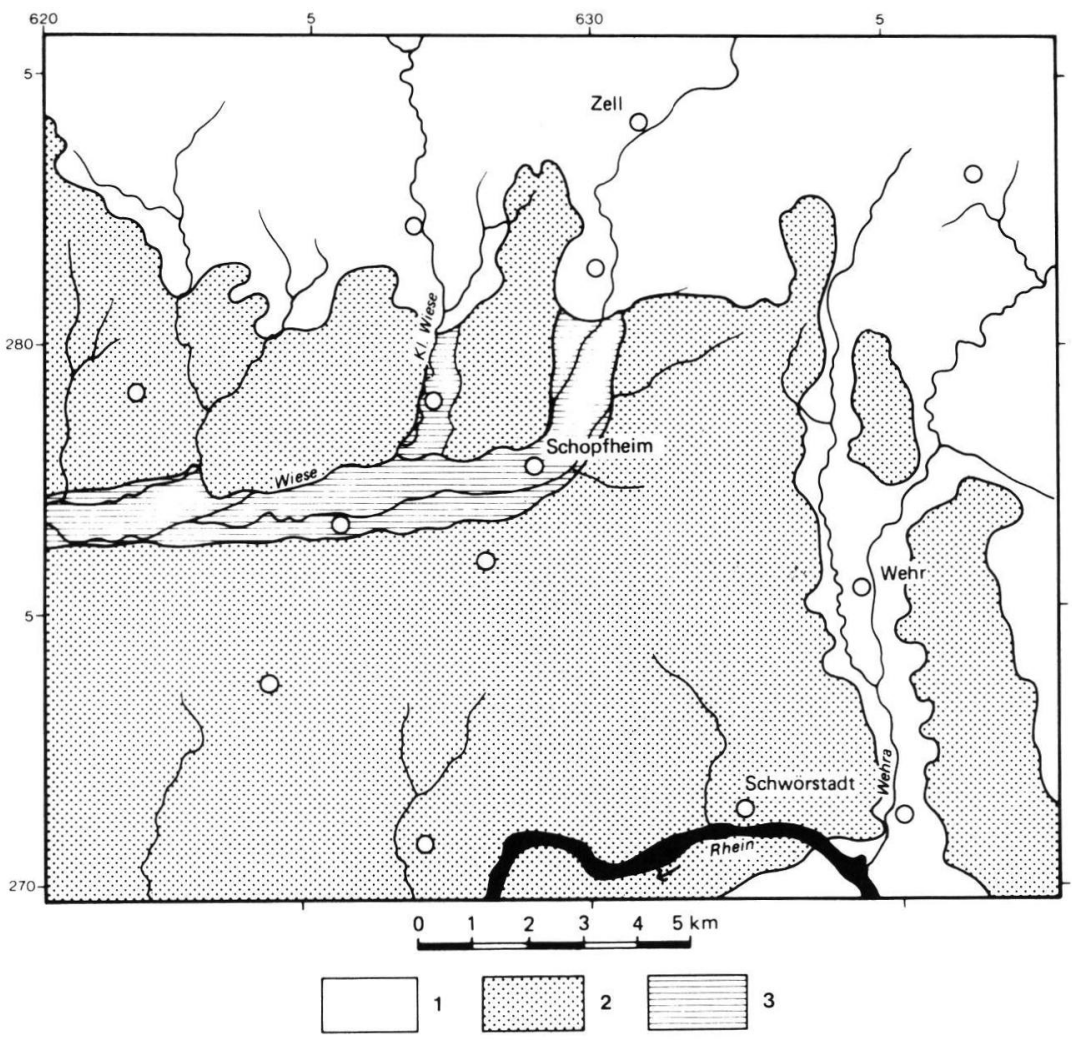

Abb. 1: Hypothese der Riß-Vereisung am Schwarzwald-Südrand,

nach den Kartierungen auf Blatt Freiburg-Süd der GMK 100 sowie auf Blatt Wehr der GMK 25.

1 - Eisüberdeckung während des Maximalstandes im Riß; 2 - Periglazialgebiet; 3 - Sohlentäler von Kleiner und Großer Wiese als denkbare Abflußleitlinien der Schmelzwässer des Schwarzwaldeises.

Aus den Abbildungen 3 und 4 resultiert die hypothetische Verbreitung des Riß-Eises im Wehratal und um die Vereini-

man nicht glazigen, etwa als moränale Sedimente, erklären. Zudem setzen Flußterrassen und Schotterverbreitung auf der Dinkelberg-Hochfläche aus. Der auf der Dinkelberg-Hochfläche ausgeprägt vorhandene $\mathrm{K}$ arstformens chat z (siehe GMK 100-Blatt Freiburg-Süd und GMK 25-Blatt Wehr) hätte wenigstens teilweise zerstört werden müssen, wenn eine Eisüberfahrung erfolgt wäre. Bei dieser wird in der Literatur im übrigen von einigen Zehner Metern Mächtigkeit gesprochen.

Macht man sich Gedanken über die $G$ e s t a 1 t ung des rißzeitlichen Schwarzw a ld e i s-S ü d r a n des und unterstellt eine vollständige Vergletscherung des Schwarzwaldes während des Riß, läßt sich aus dem Formenkontext am Südrand des Schwarzwaldes allenfalls s p e k u la - gungsstelle. Interpretiert man den Formenschatz zwischen Schwarzwald und Weitenauer Bergland, ergibt sich eine Begrenzung des Riß-Eises auf die Talräume zwischen Schwarzwald und den Vorbergen des Weitenauer Berglandes. Eindeutig scheinen die breiten, mit Schotter aufgefüllten Täler der Kleinen und Großen Wiese, über welche sich Schmelzwasserflüsse vollzogen.

t i v die in Abb. 1 dargestellte Situation ableiten. Man könnte die Ausraumzonen der Kleinen und Großen Wiese als Bereiche von Gletscherzungenenden interpretieren, ebenso den Talknoten von Schlächtenhaus und Weitenau. Die breiten Sohlentäler wären demzufolge Abflußbahnen des Schmelzwassers der Gletscher der Großen und Kleinen Wiese. Die breiten Talsohlen könnten auf Seitenerosion bzw. breit mäandrierende Schmelzwasserbäche im Gletschervorfeld interpretiert werden.

Gestützt wird diese Spekulation von der begrenzten rißzeitlichen Schwarzwaldvereisung durch die Formen und Sedimente im Wehratal, über die an anderer Stelle bereits berichtet wurde (LESER 1980), 1981 a, $1981 \mathrm{~b})$. Der Gesamtformenschatz dazu findet sich im GMK 25-Blatt Wehr (LESER 1979a, 1979b) dargestellt. 


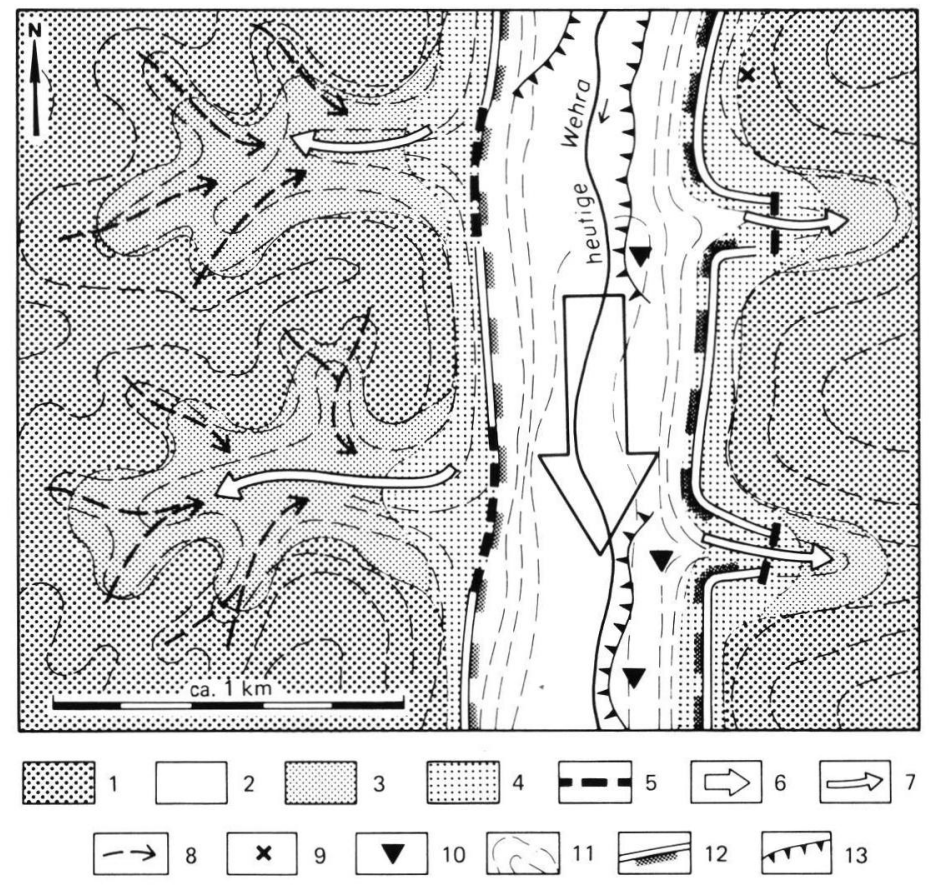

Abb. 2: Modell der Riß-Vereisung im Unteren Wehratal nördlicher Einmündung in das Hochrheintal.

1 - Periglazialgebiet;

2 - Haupteis im Wehratal;

3 - Eisloben in "hängenden"

Nebentälchen des Wehratales;

4 - Eisrand des Haupteises/

"Trogschulterbereiche";

5 - Stufenmündungen der „Hängetälchen”;

6 - Strömungsrichtung des Haupteises in Richtung Rheingletscher im Hochrheintal;

7 - zeitweiser Eisvorstoß kleiner Seitenloben;

8 - Zentripetale Entwässerung periglazialer Tälchen;

9 - Fundstelle randglazialer

Sedimente;

10 - Fundstellen von Grundmoränensedimenten auf rißzeitlichen Hochterrassenschottern; 11 - Gefühlsisohypsen des Geländes;

12 - heutige Talrandstufen nach der GMK 25-Blatt Wehr;

13 - Kante der Riß-Terrasse des heutigen Wehratales.

Das Modell geht von einem Hauptgletscher im Tal aus, von dem zeitweise kleinere Vorstöße auf den Großen Dinkelberg (links der Wehra) und den Kleinen Dinkelberg (rechts der Wehra) erfolgten. Formen und der Fund eines randglazialen Sedimentes weisen auf die mögliche Gültigkeit dieser Hypothese hin.

Der Hauptgletscher im Wehratal ist durch mehrfache Grundmoränenfunde belegt.

Aus diesen Befunden wird die $\mathrm{z}$ we it e $\mathrm{Hy}$ p othe se abgeleitet (Abb. 2): Die $\mathrm{riBz}$ e i t liche Vorlandvergletscherung des Schwarzwaldes war im Be reich des Wehratales auf das e ige nt liche Tal begrenzt und setzte an den Höhen des Großen und des Kleinen Dinkelberges - beiderseits des Wehratales - aus. Darauf weist der gesamte Formenkontext der Talränder hin, ebenso das völlige Fehlen glazigener Sedimente außerhalb der "Schultern” des Wehratales. Das in Abb. 2 dargestellte Modell der Talvergletscherung der Wehra präsentiert diese Möglichkeit der Eisverbreitung, die punktuell sedimentologisch belegt ist.

Abgestützt wird diese Überlegung durch die Befunde auf dem $\mathrm{M}$ öh li n e r F e ld, also in der Rheinschlinge zwischen Rheinfelden (Schweiz) und Wallbach (Schweiz). Die hier durchgeführten sedimentologischen und geomorphologischen Untersuchungen erlaubten eine genaue, großmaßstäbige $\mathrm{F}$ e s t legung der rißzeitlichen End $\mathrm{m}$ or ä $\mathrm{n}$ e $\mathrm{n}$, die in der älteren Glazialliteratur des
Hochrheintales schon mehrfach beschrieben wurden. Die Literatur stellt im übrigen den Eiskontakt nicht in Frage. Vielmehr wird er als sehr breit dargestellt. Dies wiederum gründet sich auf die Vorstellung von PFANNENSTIEL \& RAHM (1964), daß die gesamte Dinkelberg-Hochfläche - bis an den Abfall zum Hochrheintal - vergletschert gewesen sein muß (Abb. 3). Über die Gestaltung dieses Eisrandes wird jedoch nichts ausgesagt. Insbesondere würde interessieren, weshalb auf den Randhöhen des Dinkelberges gegen das Hochrheintal hin relativ unversehrte Jüngere Deckenschotter (eventuell auch Ältere Deckenschotter) lagern. Sie hätten, unmittelbar vor einer mehrere Zehner Meter mächtigen Eismasse, umgelagert oder eingebettet werden müssen. Die Vorkommen, z. B. auf dem Humbel (Dinkelberg-Südostecke, oberhalb der Wehratalmündung in das Hochrheintal) weisen vielmehr auf eine ungestörte, durch keinerlei glaziale Einflüsse veränderte Lagerung und Verwitterungszustände hin.

Geht man nun von der Hypothese des eiserfüllten Wehratales aus (Abb. 2), ebenso von einer eisfreien 


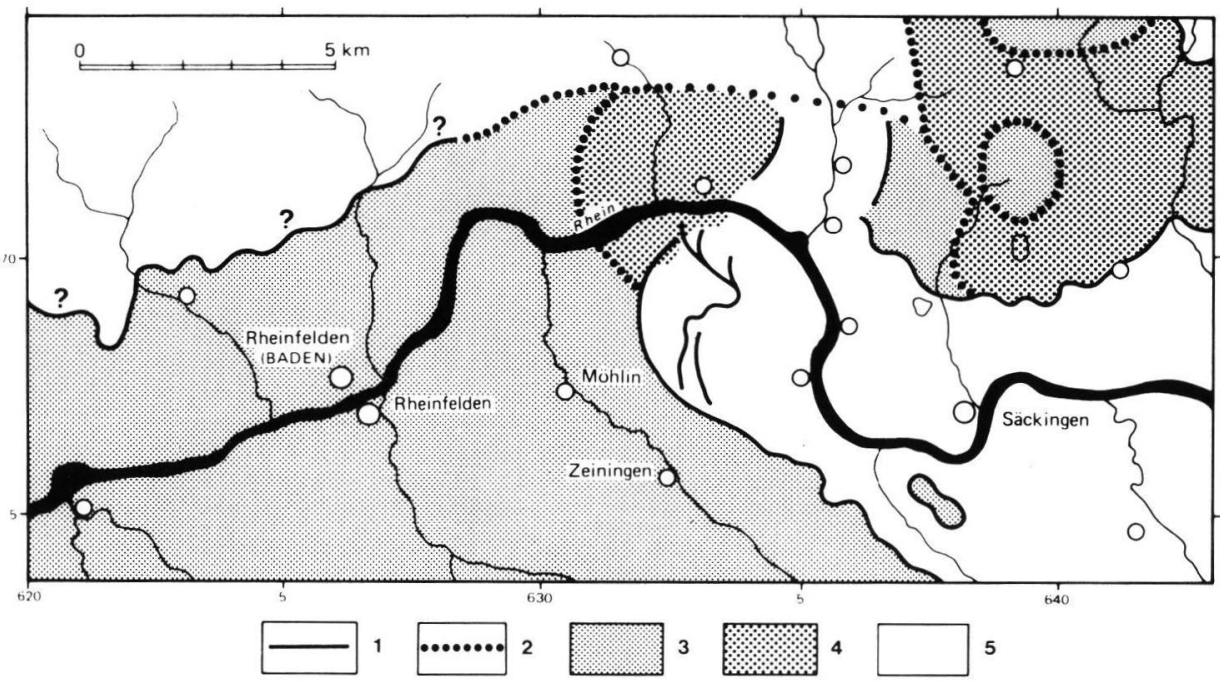

Abb. 3: Das Problem der Vereinigung des Schwarzwald- und des Alpeneises während der Riß-Kaltzeit im Bereich der Mündung des Wehratales in den Hochrhein.

1 - Maximale Vergletscherung (Riß) nach R. HANTKE (1978); 2 - Maximale Vergletscherung (Riß) nach PFANNENSTIEL \& RAHM (1976) sowie HaNTKE (1978); 3 - Während der größten Vergletscherung eisfreie Gebiete; 4 - Während der größten Vergletscherung eisfreies Areal nach PFANNEN-
STIEL \& RAHM; 5 - Eisverbreitung nach HANTKE (1978) sowie Pfannenstiel \& Rahm (1964). Das Eis und die Vereinigungsstelle liegen we s t li c h von Schwörstadt bzw. deutlich westlich der Wehramündung in den Rhein.

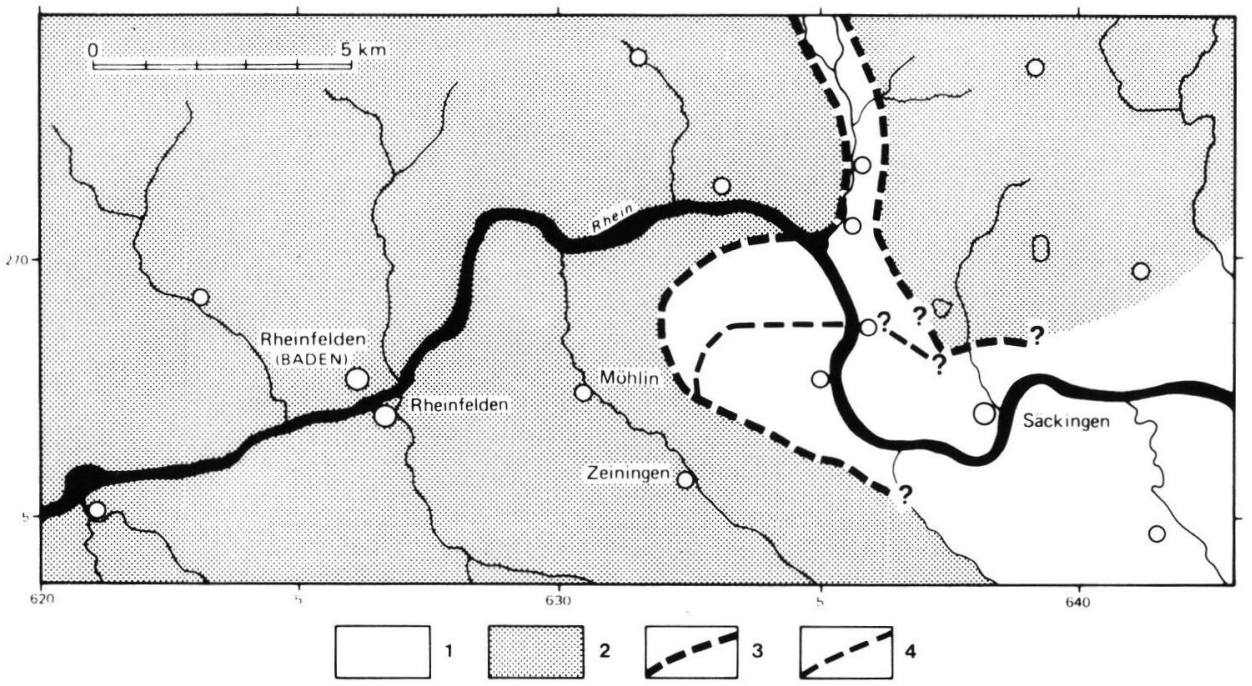

Abb. 4: Wehra- und Rhein-Gletscherkontakt (hypothetisch) zur Zeit des Maximalstandes im Riß.

1 - Eisverbreitung im Hochrhein- und Wehratal zur Zeit des Maximalstandes; 2 - Eisfreies Gebiet zur Zeit des Maximalstandes; 3 - Maximale Eisverbreitung im Wehratal und auf dem Möhliner Feld; 4 - Weitaus schwächerer Vorstoß nach dem Maximalvorstoß, wobei der Eiskontakt zum Wehratal nicht mehr bestand. Das Verbreitungsmuster der Endmoränenbögen auf dem Möhliner Feld hat zur
Konsequenz, daß die Vereinigung von Schwarzwald- und Alpeneis relativ kurz war und offensichtlich nur während des Maximalstandes bestehen konnte. Ansonsten müßte die Westflanke des Unteren Wehratales (Gebiet des Humbel und der südöstlichsten Dinkelbergecke) einen entsprechenden Formenschatz sowie eine einschlägige Sedimentverbreitung erkennen lassen. 
Dinkelberg-Hochfläche, bekommt der neu bestimmte r i z e it liche Endmoränenverlauf a u f d e m M öh lin e r F e ld mit einem Male einen Sinn. Die Bögen ziehen nämlich nicht, wie in manchen Karten in der Literatur dargestellt (Abb. 3), in Richtung Schwörstadt, also nach N. Sondern der äußere Bogen weist mit seinem Nordflügel ,zurück” in Richtung Wehratalmündung, während der innere Bogen sogar eine noch kleinere Zunge des Rheingletschers im Hochrheintal belegt, welche den Wehratalausgang gar nicht mehr erreichen konnte (Abb. 4).

Auch hier spricht der gesamte Formenkontext für eine begrenztere, dafür aber um so realistischere rißzeitliche Eisverbreitung. Das bedeutet, daß sich der rißzeitliche Hochrhein-Gletscher allenfalls zu Zeiten des $M$ a i malstandes in einem Eis $\mathrm{k}$ o n t a k t mit dem Schwarzwaldeis befand. Bereits der schwächere, dahinterliegende Vorstoß erreichte das Wehratalgletscherende nicht mehr. Er dürfte im Bereich seines Vorderendes ausgesprochen dünn und geringmächtig gewesen sein, weil Glazialspuren im Formenschatz nicht auffindbar sind. Lediglich die Grundmoräne auf Terrassenschotter bei Öflingen/Brennet (LESER 1981 b), wenig nördlich der Wehramündung in das Hochrheintal, weist eindeutig darauf hin, daß sich das Schwarzwaldeis des Wehragletschers bis zur Nahtstelle zum Alpeneis des Hochrheingletschers vorbewegt haben muß.

\section{Schriftenverzeichnis}

Fränzle, O., Barsch, D., Leser, H., LiedtKe, H. \& StäBLEIN, G. (1979): Legendenentwurf für die geomorphologische Karte 1:100000. GMK 100. - Heidelberger Geogr. Arbeiten, 65: 1-18, Heidelberg.

HANTKE, R. (1978): Eiszeitalter. Bd. 1: Die jüngste Erdgeschichte der Schweiz und ihrer Nachbargebiete. Klima, Flora, Fauna, Mensch, Alt- und Mittel-Pleistozän, Vogesen, Schwarzwald, Schwäbische Alb, Adelegg. 1-468, Thun (Ott.).

KÜHNEN, H. (1983): Geomorphologische Kartierung im Maßstab 1:10000 auf dem mittleren Möhliner Feld (Rheinschlinge zwischen Wallbach und Rheinfelden, Schweiz) mit besonderer Berücksichtigung der Reliefund Substratgenese im Pleistozän und Holozän. Diplomarbeit Geogr. Inst. Universität Basel: 1-249; Basel. - [Unveröff.]

- (1984): Sedimente und Reliefformen auf dem Möhliner Feld. - Regio Bas., XXV: 3-9; Basel.
LESER, H. (1979 a): Geomorphologische Karte der Bundesrepublik Deutschland 1:25000. Blatt 4: 8313 Wehr; Berlin.

- (1979b): Erläuterung zur Geomorphologischen Karte $1: 25000$ der Bundesrepublik Deutschland. GMK 25 Blatt 4: 8313 Wehr. - 1-60; Berlin.

- (1980): Zum Problem rißzeitlicher Sedimente im Wehra-Tal (Südschwarzwald). - Oberrhein. Geol. Abh., 29: 59-69; Karlsruhe.

- (1981 a): Ein randglaziales Sediment aus der Rißkaltzeit bei Wehr (Südschwarzwald). - Eiszeitalter u. Gegenwart, 31: 23-36; Stuttgart.

- (1981 b): Eine rißzeitliche Grundmoränenablagerung in Öflingen/Brennet im Wehratal (Südschwarzwald). Jb. geol. L.-Amt Baden-Württemberg, 23: 15-43; Freiburg / Br.

- (1985 a): Geomorphologische Karte der Bundesrepublik Deutschland 1:100000. Blatt 2, C 8310 Freiburg-Süd. - Berlin.

- (1985 b): Erläuterungen zur Geomorphologischen Karte 1: 100000 der Bundesrepublik Deutschland. Blatt 2, C 8310 Freiburg-Süd. - 1-94, Berlin.

- \& B. METZ [o. J.]: Vergletscherungen im Hochschwarzwald. - Berliner Geogr. Abh. = Beiträge zum GMKSchwerpunktprogramm VIII, ca. 25 S., Berlin.

- \& G. STÄBLEIN (1978): Legende der Geomorphologischen Karte $1: 25000$ (GMK 25). 3. Fassung im GMKSchwerpunktprogramm. - Berliner Geogr. Abh., 30: 79-90; Berlin.

Pfannenstiel, M. \& Rahm, G. (1964): Die Vergletscherung des Wehratales und der Wiesetäler während der Rißeiszeit. - Ber. Naturf. Ges. Freiburg i. Br., 54: 209-278; Freiburg/Br.

- \& - (1975): Die rißzeitliche Vergletscherung des Blauen bei Badenweiler. - Ber. Naturf. Ges. Freiburg i. Br., 65: 81-96; Freiburg / Br.

RAHM, G. (1970): Die Vergletscherung des Schwarzwaldes im Vergleich mit denjenigen der Vogesen. - Alem. Jb. 1966/67: 257-272; Freiburg i. Br.

- (1980): Die ältere Vereisung des Schwarzwaldes und der angrenzenden Gebiete. - In: LIEHL, E. \& SICK, W.-D. (Hg.) 1980: Der Schwarzwald. Beiträge zur Landeskunde: 36-58; Bühl/Baden.

Manuskript eingegangen am 15. 3. 87. 